\title{
Elastografía en tiempo real (Supersonic), experiencia de un centro en Bogotá
}

\section{Real-time elastography (Supersonic), experience of a medical center in Bogotá}

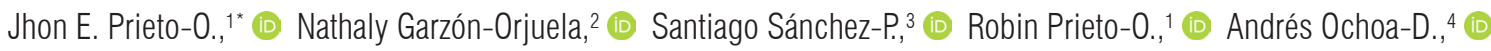 \\ Pedro H. Soto-0., ${ }^{1}$ (D) Javier Eslava-Schmalbach. ${ }^{5}$ (D)
}

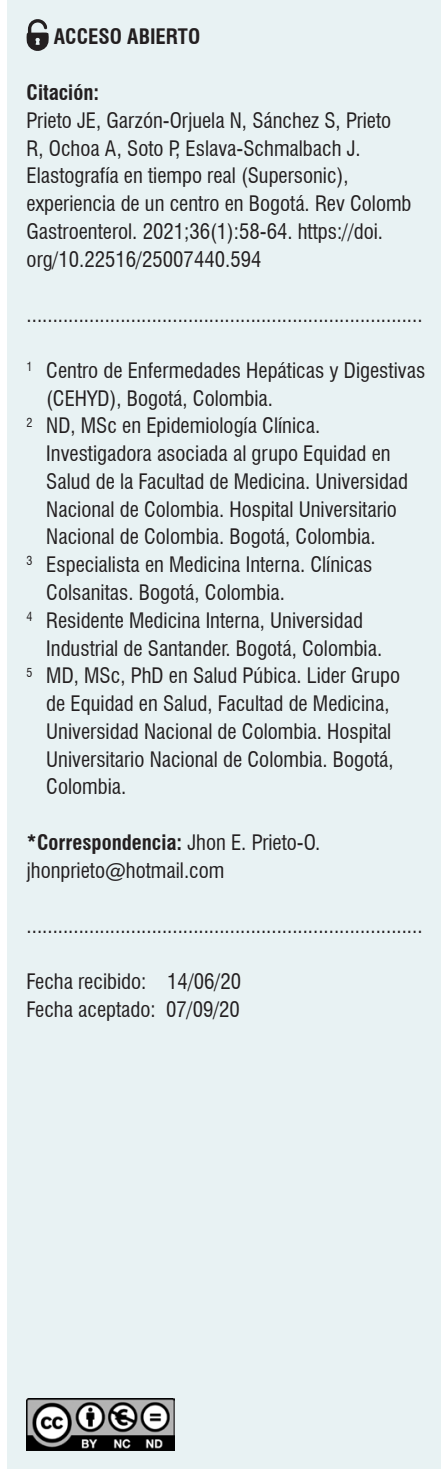

\section{Resumen}

Introducción: la elastografía en tiempo real, 2D-SWE (Supersonic), es una prueba no invasiva que se utiliza para determinar la elasticidad del hígado y, de esa forma, calcular el grado de fibrosis hepática. En Colombia, la prueba se introdujo en 2016 y no existen hasta el momento estudios del comportamiento de la prueba en todos los pacientes hepáticos, solo se han publicado en pacientes sanos y cirróticos. Objetivo: analizar la experiencia de la aplicación de la elastografía en tiempo real, en sujetos atendidos en el centro de enfermedades hepáticas y digestivas de Bogotá, Colombia. Materiales y métodos: estudio descriptivo retrospectivo de una cohorte de sujetos atendidos entre marzo de 2016 y julio de 2017. Se realizó una historia clínica completa y una prueba de elastografía en tiempo real (Supersonic). Resultados: se incluyeron 654 sujetos, con una mediana de edad de 55 años (rango intercuartílico [RIC]: 45-64). La mediana de valores de fibrosis expresada en kilopascales (kPs) fue de 8,3, con un promedio de 5 mediciones. Se observó una diferencia significativa en el grado de fibrosis entre los grupos de edad y en relación con el diagnóstico final, donde se evidenció una mayor fibrosis en el grupo de enfermedades colestásicas (autoinmune, colangitis biliar primaria [CBP] y superposición autoinmune-CBP). La tasa global de fracaso fue menor al $1 \%$. Conclusiones: es la primera descripción del comportamiento de la prueba a nivel nacional. Los valores de rigidez hepática observados en los diferentes estadios demuestran la utilidad de la prueba para la determinación de la fibrosis hepática en pacientes con diferentes patologías.

\section{Palabras clave}

2D-SWE, elastografía en tiempo real, Supersonic, fibrosis hepática, evaluación no invasiva de la fibrosis hepática.

\section{Abstract}

Introduction: Real-time two-dimensional shear wave elastography (2D-SWE) (Supersonic Imagine SA), is a non-invasive test used to determine liver elasticity and calculate the degree of liver fibrosis. In Colombia, this test was introduced in 2016 and, to date, no study has tested its behavior in all liver patients, only in healthy and cirrhotic patients. Objective: To analyze the experience of real-time elastography implementation in subjects treated at the Centro de Enfermedades Hepáticas y Digestivas in Bogotá, Colombia. Materials and methods: This is a retrospective descriptive study of a cohort of subjects treated between March 2016 and July 2017. A complete medical report and a real-time elastography (Supersonic) test were performed. Results: 654 subjects were included, with a median age of 55 years (interquartile range [IQR]: 45-64). The median fibrosis values expressed in kilopascals (kPs) were 8.3, with an average of 5 measurements. There was a significant difference in the degree of fibrosis between the age groups and in relation to the final diagnosis, where there was a greater fibrosis in the cholestatic disease group (autoimmune, primary biliary cholangitis [PBC], and overlap). The overall failure rate was less than $1 \%$. Conclusions: This is the first description of this test behavior in the country. Hepatic stiffness values observed in the different stages demonstrate the usefulness of the test to establish the degree of liver fibrosis in patients with multiple diseases.

\section{Keywords}

2D-SWE, Real-time elastography, Supersonic, Liver fibrosis, Non-invasive assessment of liver fibrosis. 


\section{INTRODUCCIÓN}

Ante un paciente con enfermedad hepática crónica luego de un diagnóstico adecuado, el reto es establecer el grado de fibrosis en que se encuentra, para determinar el pronóstico y enfocar el tratamiento $(1,2)$. En la práctica clínica diaria se determina el grado de fibrosis mediante la biopsia hepática, que es la prueba de oro, utilizando el sistema de puntuación francés METAVIR, una escala de 5 puntos (de 0 a 4) en la que F0 es fibrosis ausente; F1, fibrosis periportal; F2, tabiques porta-porta iniciales con arquitectura intacta; F3, distorsión arquitectónica sin cirrosis franca; y F4, cirrosis (3). La biopsia hepática es una prueba invasiva, con errores inter- e intraobservador (4-6) y esto ha dirigido la investigación en los últimos años hacia la obtención de pruebas no invasivas que puedan cumplir el mismo papel; dentro de ellas se han desarrollado las basadas en ecografía hepática utilizando ondas de corte (7-11), una de ellas se introdujo en nuestro país a principios del 2016: la elastografía de onda de corte bidimensional (2D-SWE; Supersonic Imagine, Aixplorer ${ }^{\circledR}$, Aix-en-Provence, Francia) (12-18), conocida en nuestro medio como Supersonic; sin embargo, no disponemos hasta el momento de publicaciones que evidencien la experiencia en nuestro medio de la prueba en todo tipo de pacientes; solo se han publicado 2 estudios piloto en pacientes sanos y cirróticos en los que se buscaron validaciones de los puntos de corte respectivos (19-20). Debido a lo anterior, el objetivo de este trabajo es mostrar esta experiencia en nuestro centro con pacientes de diferentes patologías.

\section{METODOLOGÍA}

Estudio de corte descriptivo retrospectivo de pacientes que asistieron a consulta especializada de hepatología en el centro de enfermedades hepáticas y digestivas (CEHYD) en la ciudad de Bogotá. Se revisaron todas las historias clínicas de pacientes entre marzo del 2016 y julio de 2017, a los cuales se les había realizado un Supersonic, como criterio fundamental de inclusión. La prueba se realizó siguiendo el protocolo recomendado por el fabricante y utilizado en los estudios pivotales publicados (13-16) y previamente mencionado en nuestras publicaciones (19-20) así: sistema de ultrasonido Aixplorer (Supersonic Imagine S.A. Aixen-Provence, Francia) con una sonda convexa de banda ancha (SC6-1). El software del equipo codifica los datos de elasticidad por colores, que crea un mapa bidimensional de rigidez tisular mostrado en un recuadro, allí se ubica una región de interés (ROI), en la que se mide la rigidez hepática; se utiliza un promedio de $1,5 \mathrm{~cm}$ de ROI y un recuadro de 3,5 $\times 2 \mathrm{~cm}$. El examen se realizó con el paciente en ayunas, acostado en posición supina con el brazo dere- cho en la abducción máxima, las mediciones se realizaron en el lóbulo derecho del hígado, a través de los espacios intercostales. Se consideraron solo las elastografías con 5 o más mediciones adecuadas. En nuestra serie se utilizan los puntos de corte del estudio de Ferraioli en su estudio de pacientes con hepatitis $\mathrm{C}$, que son los más utilizados (14).

De acuerdo con la Resolución 8430 de 1993 del Ministerio de la Protección Social, este estudio corresponde a una investigación sin riesgo y cuenta con la autorización de la institución en la que se atendieron los pacientes.

\section{Análisis estadístico}

La información recolectada fue resumida descriptivamente, las variables cualitativas se presentaron en frecuencias absolutas y las variables cuantitativas, mediante medidas de tendencia central y de dispersión, dependiendo de su distribución normal (prueba de Shapiro-Wilk), y prueba de la U de Mann-Whitney y prueba de Kruskal-Wallis.

\section{RESULTADOS}

En el período del estudio comprendido entre marzo del 2016 y julio de 2017 se incluyó una cohorte de 654 sujetos de 15 a 90 años de edad, la mayoría en estudio para enfermedades hepáticas, y un subgrupo de individuos sanos. En toda la cohorte, la mediana de edad fue de 55 años con un rango intercuartílico (RIC), de 45-64 en mujeres y un RIC de 40-63 en hombres, el $62 \%$ de todos los sujetos incluidos fueron mujeres. En la Tabla 1 se detallan características clínicas y de laboratorio según el sexo.

En relación con el comportamiento de la prueba, la mediana de valores de rigidez hepática expresada en kilopascales (kPs) y relacionada directamente con el grado de fibrosis hepática fue de 8,3 , con un promedio de 5 mediciones y sin diferencias por sexo. Se observó una diferencia significativa en la rigidez hepática entre los grupos de edad, con una clara tendencia a tener valores mayores a mayor edad.

En cuanto al diagnóstico final, se evidenció una mayor rigidez (fibrosis), en el grupo de enfermedades colestásicas (autoinmune, colangitis biliar primaria $[\mathrm{CBP}]$ y superposición autoinmune-CBP), con una mediana de $9,7 \mathrm{kPs}$, seguido por el grupo de las virales (virus $\mathrm{C}$ y B) y el hígado graso. 25 individuos presentaron una rigidez hepática mínima, con una mediana de $4,4 \mathrm{kPs}$, lo que sugiere una fibrosis cero y se consideraron sanos. Los datos completos se muestran en la Tabla 2. Finalmente, en la Figura 1 se observa el comportamiento de la prueba al expresar los valores de rigidez hepática como estadios de fibrosis de la escala METAVIR (utilizando los puntos de corte del estudio pivotal de Ferraioli) (14), que mostró una diferencia significativa en su clasificación y en las medianas de fibrosis (kPs). 
Tabla 1. Características clínicas y de laboratorio

\begin{tabular}{|c|c|c|c|c|}
\hline \multirow[t]{2}{*}{ Característica } & \multicolumn{2}{|r|}{ Mujeres } & \multicolumn{2}{|r|}{ Hombres } \\
\hline & n & Mediana (RIC)* & $\mathbf{n}$ & Mediana (RIC)* \\
\hline Edad en años & 406 & $56(45-64)$ & 248 & $52(40-63)$ \\
\hline IMC & 354 & $25(22-28)^{ \pm}$ & 197 & $26(23-28)^{ \pm}$ \\
\hline Leucocitos (cel/mL) & 362 & $5440(4580-6600)$ & 199 & $5710(4900-6800)$ \\
\hline $\mathrm{Hb}(\mathrm{g} / \mathrm{dL})$ & 362 & $14(13-15)^{ \pm}$ & 198 & $16(15-17)^{ \pm}$ \\
\hline Hto (\%) & 362 & $43(40-45)^{ \pm}$ & 199 & $48(45-50)^{ \pm}$ \\
\hline Plaquetas (cel/mm³) & 362 & $250000(196000-303000)^{ \pm}$ & 199 & $210000(169000-254000)^{ \pm}$ \\
\hline Glucemia (mg/dL) & 355 & $90(83-97)^{ \pm}$ & 195 & $94(87-102)^{ \pm}$ \\
\hline AST (UI/dL) & 364 & $40(24-78)$ & 200 & $36(25-59)$ \\
\hline ALT (UI/dL) & 364 & $46(25-92)$ & 200 & $51(29-103)$ \\
\hline GGT (UI/dL) & 351 & $60(25-184)$ & 192 & $62(34-146)$ \\
\hline Fosfatasa alcalina (UI/dL) & 362 & $106(77-184)^{ \pm}$ & 194 & $90(72-121)^{ \pm}$ \\
\hline Bilirrubina total (mg/dL) & 355 & $0,6(0,4-1,0)^{ \pm}$ & 189 & $0,7(0,6-1,2)^{ \pm}$ \\
\hline Proteínas totales (g/dL) & 340 & $7,3(7-7,8)$ & 185 & $7,3(7-7,7)$ \\
\hline Albúmina (g/dL) & 348 & $4,3(4-4,5)^{ \pm}$ & 183 & $4,4(4,1-4,7)^{ \pm}$ \\
\hline
\end{tabular}

*Prueba de normalidad (Shapiro-Wilk). \pm Diferencias significativas entre mujeres y hombres $(\mathrm{p}<0,05)$, prueba de la U de Mann-Whitney. ALT: alanina-aminotransferasa; AST: aspartato-aminotransferasa; GGT: $\gamma$-glutamiltransferasa; Hb: hemoglobina; Hto: hematocrito; IMC: índice de masa corporal; RIC: rango intercuartílico (percentil 25 y 75 ).

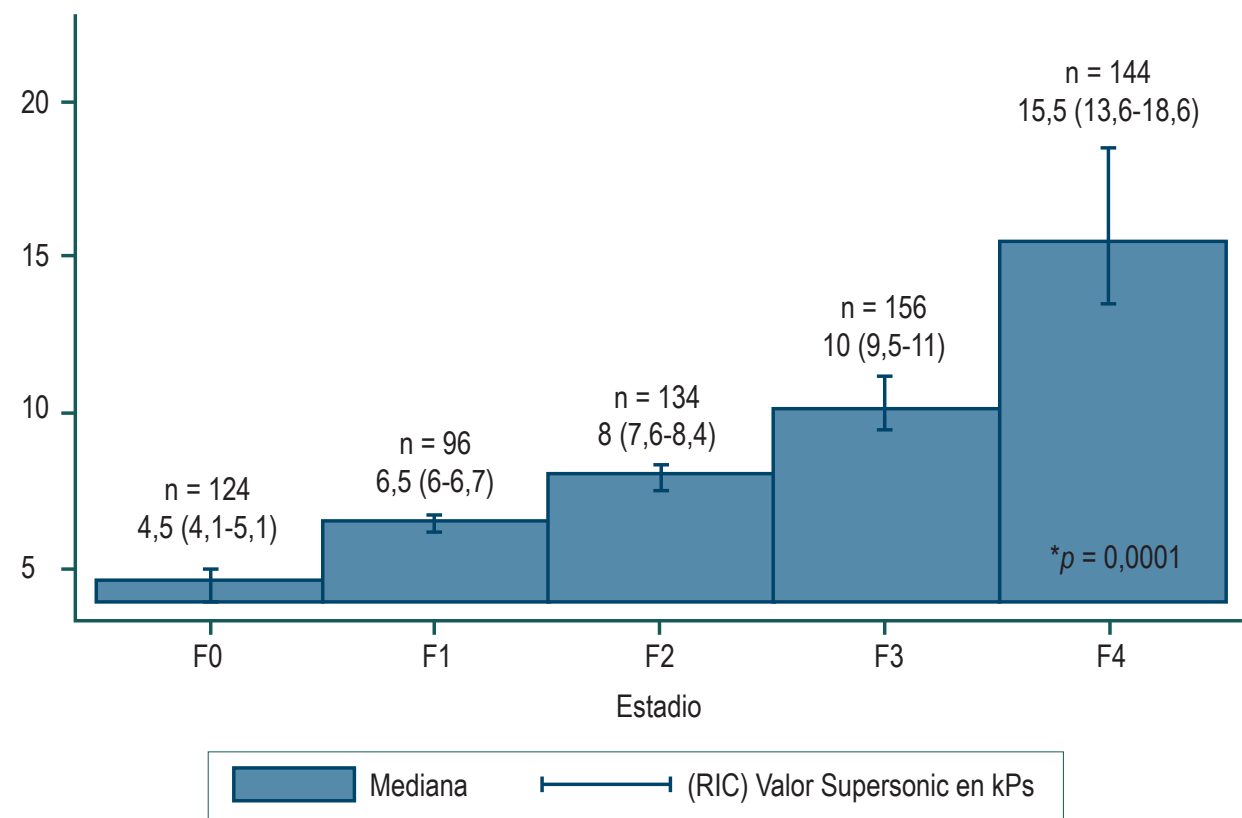

Figura 1. Estadios de fibrosis obtenidos con el Supersonic. ${ }^{*}$ Diferencias significativas entre los grupos de estadios, prueba de Kruskal-Wallis. 
Tabla 2. Comportamiento del Supersonic según el sexo, la edad y la hepatopatía

\begin{tabular}{|c|c|c|c|}
\hline Variable & $n(\%)$ & $\begin{array}{c}\text { Mediana } \\
(\text { RIC)* valores } \\
\text { en kPs }\end{array}$ & $\begin{array}{c}\text { Mediana } \\
\text { (RIC)*, } \\
\text { cantidad de } \\
\text { mediciones }\end{array}$ \\
\hline Total de sujetos & 654 & $8,3(6,4-11,5)$ & $5(5-6)$ \\
\hline \multicolumn{4}{|l|}{ Sexo } \\
\hline - Mujeres & $406(62,1)$ & $8,3(6,3-11,7)$ & $5(5-6)$ \\
\hline - Hombres & $248(37,9)$ & $8,3(6,5-11,9)$ & $5(5-6)$ \\
\hline Edad ** & & ** & \\
\hline$-\leq 20$ & $23(3,5)$ & $5(4,4-5)$ & $5(3-6)$ \\
\hline - $21-30$ & $49(7,5)$ & $5,8(4,2-8,1)$ & $5(4-5)$ \\
\hline - $31-40$ & $70(10,7)$ & $7,6(5,5-8,9)$ & $5(5-6)$ \\
\hline$-41-50$ & $115(17,6)$ & $7,7(5,7-10,4)$ & $5(5-6)$ \\
\hline - 51-60 & $180(27,5)$ & $8,5(6,8-11,7)$ & $5(5-6)$ \\
\hline - $61-70$ & $145(22,2)$ & $9,1(7,6-12,5)$ & $5(5-6)$ \\
\hline$-\geq 71$ & $72(11)$ & $10,8(8,1-14,1)$ & $5(5-6)$ \\
\hline $\begin{array}{l}\text { Diagnóstico final de la } \\
\text { hepatopatía** }\end{array}$ & & ** & ** \\
\hline - Hígado graso & $179(44,8)$ & $8,1(6,6-10,5)$ & $5(5-6)$ \\
\hline - Colestásica & $100(25,0)$ & $9,7(7,7-13,6)$ & $5(5-6)$ \\
\hline - Viral & $82(20,5)$ & $8,6(7,4-11,8)$ & $5(5-7)$ \\
\hline - Trombosis portal & $4(1,0)$ & $6,5(4,4-8,3)$ & $5(4-5)$ \\
\hline - Sanos & $35(8,7)$ & $4,4(3,8-4,9)$ & $3(3-5)$ \\
\hline
\end{tabular}

${ }^{*}$ Prueba de normalidad (Shapiro-Wilk). ${ }^{* *} p=0,0001$, diferencias significativas entre los grupos, prueba de la U de Mann-Whitney.

En el tiempo del estudio solamente se presentaron 4 pacientes con procedimientos fallidos, todos por una obtención inadecuada de una imagen para la medición por obesidad o espacios intercostales estrechos, ninguno por incapacidad de apnea.

\section{DISCUSIÓN}

La biopsia hepática como el estándar de oro es una prueba incompleta $(5,6)$ que requiere una interpretación adecuada en el contexto individual de cada paciente, esto ha motivado la investigación de métodos no invasivos de evaluación de la fibrosis hepática que sean de uso masivo y que apoyen el estudio de los pacientes hepáticos rutinaria- mente; fruto de ello son las diferentes guías emitidas sobre los métodos no invasivos que incluso sugieren a la elastografía como método alterno a la biopsia hepática (21-23).

En Colombia, desde principios de 2016 disponemos del Supersonic o elastografía en tiempo real (2D-SWE), una prueba de fibrosis no invasiva, basada en ecografía, validada en diferentes estudios $y$, aunque varios estudios han demostrado un rendimiento superior sobre la elastografía transitoria, por lo menos es igualmente efectiva con ventajas adicionales como la visualización del hígado y la realización de la prueba en pacientes con ascitis (24-34). Este es el primer artículo que muestra la experiencia de su aplicación en nuestros pacientes y fue presentado como póster en el congreso de ACADI, 2017.

Nuestros datos muestran varios puntos a resaltar:

- Una mayor fibrosis a mayor edad; hay estudios en humanos y en animales que asocian mayor fibrosis con la edad debido al daño progresivo por el hígado graso y en nuestra serie casi una tercera parte de los pacientes tenía hígado graso; otra posible razón y tal vez más importante sería que, a mayor tiempo de una enfermedad, mayor progresión hacia cirrosis, como en caso de la hepatitis $\mathrm{C}$ y la hepatopatía por alcohol, cuyas manifestaciones aparecen luego de 20 a 40 años de enfermedad y culminan en la cirrosis (35-38).

- Se observó mayor fibrosis en el grupo de pacientes con enfermedades colestásicas (autoinmune, CBP y superposición autoinmune-CBP), seguido por el grupo de las enfermedades virales (virus C y B) y el hígado graso. En el metaanálisis de Hermann (30) se evidenció más fibrosis en los pacientes con hepatitis $\mathrm{C}$ por encima del grupo de hígado graso, al igual que nosotros, pero esta serie y otras revisadas poco mencionan cohortes de pacientes con enfermedades colestásicas; por una parte, esto podría deberse a que la mayoría de estudios se realizaron durante el boom de la hepatitis $\mathrm{C}$ y pareciera que, por otra parte, nosotros tenemos más pacientes con enfermedades colestásicas (dato a confirmar).

- Encontramos un porcentaje de procedimientos fallidos menor del $1 \%$, la mayoría presentados en los primeros meses de uso del equipo y asociados posiblemente también con la curva de aprendizaje. Leung (18) mostró una tasa de fracasos de 1,1 contra $10,4 \%$ de la elastografía transitoria; pero en pacientes con hepatitis B, Ferraioli (14) reportó una tasa de fracasos de 2,5 excluyendo ascitis, con porcentajes similares para elastografía transitoria. Castera (39), en el estudio más grande de elastografía transitoria, mostró un fracaso de $3,1 \%$ en todas las pruebas ( $4 \%$ en el primer examen, $\mathrm{n}=7261)$, más resultados no confiables en un $16 \%$ adicional; la mayoría de fracasos se debieron a obesidad, espacios intercostales estrechos e incapacidad de 
los pacientes para realizar una apnea adecuada $(13,14$, 16). La 2D-SWE o Supersonic se afecta por las mismas causas, pero menos por la obesidad, ya que la presión adicional sobre la sonda reduce el espesor de la capa grasa entre la sonda y la caja torácica, y la profundidad puede llegar a $10-12 \mathrm{~cm}$ (27-31). Adicionalmente, otro aspecto que puede influir disminuyendo la tasa de fracasos es la experiencia del operador, como lo muestran algunas series $(40,41)$.

- Una clara discriminación de nuestros pacientes en 5 grupos de pacientes de acuerdo con el grado de fibrosis (asimilándolos a la escala METAVIR de la biopsia hepática), que permite dar un pronóstico y enfocar un tratamiento en cada uno de ellos. Con respecto a los puntos de corte, nosotros utilizamos los del estudio de Ferraioli para hepatitis C (14), es decir: $\mathrm{F} \geq 2$ de 7,1, F $\geq 3$ de 8,7 y cirrosis $\geq 10,4$ kPs. Herrmann (30) utilizó para todos los pacientes cortes similares: $\mathrm{F} \geq 2$ de 7,1, $\mathrm{F}$ $\geq 3$ de 9,2 y cirrosis $\geq 13,4 \mathrm{kPs}$, que son muy similares para pacientes con hígado graso, aunque mucho mayores que en los pacientes con hepatitis B; sin embargo, en nuestra serie el número total de hepatitis B fue muy bajo. Adicional a los anteriores puntos de corte, en los estudios de Cassinotto y Sporea (12, 42), los puntos de corte de cirrosis son de 10,7 kPs y 11,5 kPs, respectivamente. En estudios recientes, el límite para hipertensión portal clínicamente significativa en pacientes con cirrosis fue establecido como $\geq 14 \mathrm{kPs}$ (43) y un valor de $10 \mathrm{kPs}$ puede ser usado para descartar la enfermedad hepática crónica avanzada compensada (cACLD); de acuerdo con los criterios de Baveno VI (44), todos estos datos nos ubican en un valor de cirrosis cercano a los 12-13 kPs, que es el límite en nuestra práctica diaria y cercano al valor que encontramos en nuestro estudio de pacientes con cirrosis, sin diferenciar, cirrosis compensada o descompensada, o su causa (20).

Somos conscientes de las limitaciones de nuestra serie, como el carácter retrospectivo y la no disponibilidad de puntos de corte propios en las diferentes entidades, pero esperamos pronto ampliar la serie, compararla con la biopsia, validar los puntos de corte en nuestros pacientes con hígado graso y con enfermedades colestásicas, importantes por sus prevalencias cerca del $40 \%$ y $10 \%$, respectivamente, en nuestra base de datos (no publicada). No obstante, se muestra por primera vez la experiencia en nuestro medio con un buen número de pacientes y resaltamos la tasa de fracaso menor del $1 \%$ en la realización de esta prueba.

\section{CONCLUSIONES}

Es la primera descripción del comportamiento de la prueba a nivel nacional, aplicando valores de rigidez hepática de los estudios pivotales. Los valores de fibrosis observados en los diferentes estadios demuestran la utilidad de la prueba para la determinación de la fibrosis hepática en pacientes con diferentes patologías con una muy baja tasa de fracasos de la prueba. Se espera ampliar el número de pacientes en series posteriores.

\section{Conflictos de interés}

Los autores declaran no tener conflictos de interés.

\section{Fuentes de financiamiento}

Con recursos propios.

\section{REFERENCIAS}

1. European Association for the Study of the Liver. EASL Clinical Practice Guidelines: management of hepatitis C virus infection. J Hepatol. 2011;55(2):245-64. https://doi. org/10.1016/j.jhep.2011.02.023

2. Ghany MG, Strader DB, Thomas DL, Seeff LB; American Association for the Study of Liver Diseases. Diagnosis, management, and treatment of hepatitis C: an update. Hepatology. 2009;49(4):1335-74. https://doi. org/10.1002/hep.22759

3. Bedossa P, Poynard T. An algorithm for the grading of activity in chronic hepatitis C. The METAVIR Cooperative Study Group. Hepatology. 1996;24(2):289-93. https://doi. org/10.1002/hep.510240201
4. Intraobserver and interobserver variations in liver biopsy interpretation in patients with chronic hepatitis $\mathrm{C}$. The French METAVIR Cooperative Study Group. Hepatology. 1994;20(1 Pt 1):15-20.

5. Soloway RD, Baggenstoss AH, Schoenfield LJ, Summerskill WH. Observer error and sampling variability tested in evaluation of hepatitis and cirrhosis by liver biopsy. Am J Dig Dis. $1971 ; 16(12): 1082-6$.

6. Bedossa $P$, Dargère $D$, Paradis V. Sampling variability of liver fibrosis in chronic hepatitis C. Hepatology. 2003;38(6):1449-57. https://doi.org/10.1016/j. hep.2003.09.022 
7. Deffieux T, Montaldo G, Tanter M, Fink M. Shear wave spectroscopy for in vivo quantification of human soft tissues visco-elasticity. IEEE Trans Med Imaging. 2009;28(3):313-22. https://doi.org/10.1109/ TMI.2008.925077

8. Sandrin L, Fourquet B, Hasquenoph JM, Yon S, Fournier C, Mal F, Christidis C, Ziol M, Poulet B, Kazemi F, Beaugrand M, Palau R. Transient elastography: a new noninvasive method for assessment of hepatic fibrosis. Ultrasound Med Biol. 2003;29(12):1705-13. https://doi.org/10.1016/j. ultrasmedbio.2003.07.001

9. Castéra L, Vergniol J, Foucher J, Le Bail B, Chanteloup E, Haaser M, Darriet M, Couzigou P, De Lédinghen V. Prospective comparison of transient elastography, Fibrotest, APRI, and liver biopsy for the assessment of fibrosis in chronic hepatitis C. Gastroenterology. 2005;128(2):343-50. https://doi.org/10.1053/j.gastro.2004.11.018

10. Friedrich-Rust M, Ong MF, Martens S, Sarrazin C, Bojunga J, Zeuzem S, Herrmann E. Performance of transient elastography for the staging of liver fibrosis: a meta-analysis. Gastroenterology. 2008;134(4):960-74. https://doi. org/10.1053/j.gastro.2008.01.034

11. Martínez SM, Crespo G, Navasa M, Forns X. Noninvasive assessment of liver fibrosis. Hepatology. 2011;53(1):32535. https://doi.org/10.1002/hep.24013

12. Cassinotto C, Lapuyade B, Mouries A, Hiriart JB, Vergniol J, Gaye D, Castain C, Le Bail B, Chermak F, Foucher J, Laurent F, Montaudon M, De Ledinghen V. Non-invasive assessment of liver fibrosis with impulse elastography: comparison of Supersonic Shear Imaging with ARFI and FibroScan ${ }^{\circledast}$ J Hepatol. 2014;61(3):550-7. https://doi. org/10.1016/j.jhep.2014.04.044

13. Bavu E, Gennisson JL, Couade M, Bercoff J, Mallet V, Fink M, Badel A, Vallet-Pichard A, Nalpas B, Tanter M, Pol S. Noninvasive in vivo liver fibrosis evaluation using supersonic shear imaging: a clinical study on 113 hepatitis C virus patients. Ultrasound Med Biol. 2011;37(9):1361-73. https://doi.org/10.1016/j.ultrasmedbio.2011.05.016

14. Ferraioli G, Tinelli C, Dal Bello B, Zicchetti M, Filice G, Filice C; Liver Fibrosis Study Group. Accuracy of real-time shear wave elastography for assessing liver fibrosis in chronic hepatitis C: a pilot study. Hepatology. 2012;56(6):2125-33. https://doi.org/10.1002/hep.25936

15. Ferraioli G, Tinelli C, Zicchetti M, Above E, Poma G, Di Gregorio M, Filice C. Reproducibility of real-time shear wave elastography in the evaluation of liver elasticity. Eur J Radiol. 2012;81(11):3102-6. https://doi.org/10.1016/j. ejrad.2012.05.030

16. Sirli R, Bota S, Sporea I, Jurchis A, Popescu A, GradinaruTascău $\mathrm{O}$, Szilaski M. Liver stiffness measurements by means of supersonic shear imaging in patients without known liver pathology. Ultrasound Med Biol. 2013;39(8):1362-7. https://doi.org/10.1016/j.ultrasmedbio.2013.03.021

17. Cha SW, Jeong WK, Kim Y, Kim MY, Kim J, Kim SY, Ryu JA, Kim TY, Sohn JH, Kim YH. Nondiseased liver stiffness measured by shear wave elastography: a pilot study. J Ultrasound Med. 2014;33(1):53-60. https://doi. org/10.7863/ultra.33.1.53

18. Leung VY, Shen J, Wong VW, Abrigo J, Wong GL, Chim AM, Chu SH, Chan AW, Choi PC, Ahuja AT, Chan HL, Chu WC. Quantitative elastography of liver fibrosis and spleen stiffness in chronic hepatitis B carriers: comparison of shear-wave elastography and transient elastography with liver biopsy correlation. Radiology. 2013;269(3):910-8. https://doi.org/10.1148/radiol.13130128

19. Prieto O JE, Sánchez P S, Prieto O RG, Garzón-Orjuela N, Eslava-Schmalbach J. Elastografía en tiempo real (supersonic): comportamiento de la prueba en sujetos sanos de Bogotá. Rev Colomb Gastroenterol. 2017;32(4):332-336. https://doi.org/10.22516/25007440.176

20. Prieto O JE, Garzón-Orjuela N, Sánchez P S, Prieto O RG Ochoa D AF, Eslava-Schmalbach J. Elastografía en tiempo real (Supersonic): comportamiento de la prueba en una cohorte de pacientes con cirrosis en un centro médico de Bogotá. Rev Colomb Gastroenterol. 2018;33(3):235-241. http://dx.doi.org/10.22516/25007440.201

21. European Association for Study of Liver; Asociacion Latinoamericana para el Estudio del Higado. EASLALEH Clinical Practice Guidelines: Non-invasive tests for evaluation of liver disease severity and prognosis. J Hepatol. 2015;63(1):237-64. https://doi.org/10.1016/j. jhep.2015.04.006

22. Friedrich-Rust M, Poynard T, Castera L. Critical comparison of elastography methods to assess chronic liver disease. Nat Rev Gastroenterol Hepatol. 2016;13(7):402-11. https://doi.org/10.1038/nrgastro.2016.86

23. Dietrich CF, Bamber J, Berzigotti A, Bota S, Cantisani V, Castera L, Cosgrove D, Ferraioli G, Friedrich-Rust M, Gilja OH, Goertz RS, Karlas T, de Knegt R, de Ledinghen V, Piscaglia F, Procopet B, Saftoiu A, Sidhu PS, Sporea I, Thiele M. EFSUMB Guidelines and Recommendations on the Clinical Use of Liver Ultrasound Elastography, Update 2017 (Long Version). Ultraschall Med. 2017;38(4):e16e47. https: / / doi.org/10.1055/s-0043-103952

24. Feng JC, Li J, Wu XW, Peng XY. Diagnostic Accuracy of SuperSonic Shear Imaging for Staging of Liver Fibrosis: A Meta-analysis. J Ultrasound Med. 2016;35(2):329-39. https://doi.org/10.7863/ultra.15.03032

25. Li C, Zhang C, Li J, Huo H, Song D. Diagnostic Accuracy of Real-Time Shear Wave Elastography for Staging of Liver Fibrosis: A Meta-Analysis. Med Sci Monit. 2016;22:134959. https://doi.org/10.12659/msm.895662

26. Procopet B, Berzigotti A, Abraldes JG, Turon F, Hernandez-Gea V, García-Pagán JC, Bosch J. Real-time shear-wave elastography: applicability, reliability and accuracy for clinically significant portal hypertension. J Hepatol. 2015;62(5):1068-75. https://doi.org/10.1016/j. jhep.2014.12.007

27. Guibal A, Renosi G, Rode A, Scoazec JY, Guillaud O, Chardon L, Munteanu M, Dumortier J, Collin F, Lefort T. Shear wave elastography: An accurate technique to 
stage liver fibrosis in chronic liver diseases. Diagn Interv Imaging. 2016;97(1):91-9. https://doi.org/10.1016/j. diii.2015.11.001

28. Zeng J, Liu GJ, Huang ZP, Zheng J, Wu T, Zheng RQ, $\mathrm{Lu}$ MD. Diagnostic accuracy of two-dimensional shear wave elastography for the non-invasive staging of hepatic fibrosis in chronic hepatitis B: a cohort study with internal validation. Eur Radiol. 2014;24(10):2572-81. https://doi. org/10.1007/s00330-014-3292-9

29. Jiang T, Tian G, Zhao Q, Kong D, Cheng C, Zhong L, Li L. Diagnostic Accuracy of 2D-Shear Wave Elastography for Liver Fibrosis Severity: A Meta-Analysis. PLoS One. 2016;11(6):e0157219. https://doi.org/10.1371/journal. pone. 0157219

30. Herrmann E, de Lédinghen V, Cassinotto C, Chu WC, Leung VY, Ferraioli G, Filice C, Castera L, Vilgrain V, Ronot M, Dumortier J, Guibal A, Pol S, Trebicka J, Jansen C, Strassburg C, Zheng R, Zheng J, Francque S, Vanwolleghem T, Vonghia L, Manesis EK, Zoumpoulis P, Sporea I, Thiele M, Krag A, Cohen-Bacrie C, Criton A, Gay J, Deffieux T, Friedrich-Rust M. Assessment of biopsy-proven liver fibrosis by two-dimensional shear wave elastography: An individual patient data-based metaanalysis. Hepatology. 2018;67(1):260-272. https://doi. org/10.1002/hep.29179

31. Deng H, Qi X, Zhang T, Qi X, Yoshida EM, Guo X. Supersonic shear imaging for the diagnosis of liver fibrosis and portal hypertension in liver diseases: a meta-analysis. Expert Rev Gastroenterol Hepatol. 2018;12(1):91-98. https://doi.org/10.1080/17474124.2018.1412257

32. Cassinotto C, Boursier J, de Lédinghen V, Lebigot J, Lapuyade B, Cales P, Hiriart JB, Michalak S, Bail BL, Cartier V, Mouries A, Oberti F, Fouchard-Hubert I, Vergniol J, Aubé C. Liver stiffness in nonalcoholic fatty liver disease: A comparison of supersonic shear imaging, FibroScan, and ARFI with liver biopsy. Hepatology. 2016;63(6):1817-27. https://doi.org/10.1002/hep.28394

33. Yoneda M, Thomas E, Sclair SN, Grant TT, Schiff ER. Supersonic Shear Imaging and Transient Elastography With the XL Probe Accurately Detect Fibrosis in Overweight or Obese Patients With Chronic Liver Disease. Clin Gastroenterol Hepatol. 2015;13(8):1502-9.e5. https://doi. org/10.1016/j.cgh.2015.03.014

34. Poynard T, Munteanu M, Luckina E, Perazzo H, Ngo Y, Royer L, Fedchuk L, Sattonnet F, Pais R, Lebray P, Rudler M, Thabut D, Ratziu V. Liver fibrosis evaluation using realtime shear wave elastography: applicability and diagnostic performance using methods without a gold standard. J Hepatol. 2013;58(5):928-35. https://doi.org/10.1016/j. jhep.2012.12.021

35. Poynard T, Lebray P, Ingiliz P, Varaut A, Varsat B, Ngo Y, Norha P, Munteanu M, Drane F, Messous D, Bismut FI, Carrau JP, Massard J, Ratziu V, Giordanella JP. Prevalence of liver fibrosis and risk factors in a general population using non-invasive biomarkers (FibroTest). BMC
Gastroenterol. 2010;10:40. https://doi.org/10.1186/1471230X-10-40

36. Mahrouf-Yorgov M, Collin de l'Hortet A, Cosson C, Slama A, Abdoun E, Guidotti JE, Fromenty B, Mitchell C, Gilgenkrantz $\mathrm{H}$. Increased susceptibility to liver fibrosis with age is correlated with an altered inflammatory response. Rejuvenation Res. 2011;14(4):353-63. https://doi. org/10.1089/rej.2010.1146

37. Scaglione S, Kliethermes S, Cao G, Shoham D, Durazo R, Luke A, Volk ML. The Epidemiology of Cirrhosis in the United States: A Population-based Study. J Clin Gastroenterol. 2015;49(8):690-6. https://doi. org/10.1097/MCG.0000000000000208

38. Guarner V, Rubio-Ruiz ME. Low-grade systemic inflammation connects aging, metabolic syndrome and cardiovascular disease. Interdiscip Top Gerontol. 2015;40:99-106. https://doi.org/10.1159/000364934

39. Castéra L, Foucher J, Bernard PH, Carvalho F, Allaix D, Merrouche W, Couzigou P, de Lédinghen V. Pitfalls of liver stiffness measurement: a 5-year prospective study of 13,369 examinations. Hepatology. 2010;51(3):828-35. https:// doi.org/10.1002/hep.23425

40. Wang H, Zheng P, Sang L, Wang X. Does Operator Experience and the Q-Box Diameter Affect the Repeatability of Liver Stiffness Measurements Obtained by 2 -Dimensional Shear Wave Elastography? J Ultrasound Med. 2020;39(4):741-747. https://doi.org/10.1002/ jum. 15153

41. Grădinaru-Taşcău $O$, Sporea I, Bota $S$, Jurchiş A, Popescu A, Popescu M, Şirli R, Szilaski M. Does experience play a role in the ability to perform liver stiffness measurements by means of supersonic shear imaging (SSI)? Med Ultrason. 2013;15(3):180-3. https://doi.org/10.11152/ mu.2013.2066.153.ogt1is2

42. Sporea I, Bota S, Gradinaru-Taşcău O, Sirli R, Popescu A, Jurchiş A. Which are the cut-off values of 2D-Shear Wave Elastography (2D-SWE) liver stiffness measurements predicting different stages of liver fibrosis, considering Transient Elastography (TE) as the reference method? Eur J Radiol. 2014 Mar;83(3):e118-22. https: / / doi. org/10.1016/j.ejrad.2013.12.011

43. Thiele M, Hugger MB, Kim Y, Rautou PE, Elkrief L, Jansen C, Verlinden W, Allegretti G, Israelsen M, Stefanescu H, Piscaglia F, García-Pagán JC, Franque S, Berzigotti A, Castera L, Jeong WK, Trebicka J, Krag A. 2D shear wave liver elastography by Aixplorer to detect portal hypertension in cirrhosis: An individual patient data metaanalysis. Liver Int. 2020;40(6):1435-1446. https:// doi. org/10.1111/liv.14439

44. Cassinotto C, Lapuyade B, Guiu B, Marraud des Grottes H, Piron L, Merrouche W, Irles-Depe M, Molinari N, De Ledinghen V. Agreement Between 2-Dimensional Shear Wave and Transient Elastography Values for Diagnosis of Advanced Chronic Liver Disease. Clin Gastroenterol Hepatol. 2020;18(13):2971-2979.e3. https://doi. org/10.1016/j.cgh.2020.04.034 